\title{
METHYLENE BLUE IMPROVES MITOCHONDRIAL RESPIRATION AND DECREASES OXIDATIVE STRESS IN A SUBSTRATE-DEPENDENT MANNER IN DIABETIC RAT HEARTS
}

\begin{tabular}{|c|c|}
\hline Journal: & Canadian Journal of Physiology and Pharmacology \\
\hline Manuscript ID & cjpp-2017-0074.R1 \\
\hline Manuscript Type: & Article \\
\hline Date Submitted by the Author: & 07-Mar-2017 \\
\hline Complete List of Authors: & $\begin{array}{l}\text { Duicu, Oana; University of Medicine and Pharmacy Victor Babes Timisoara, } \\
\text { Pathophysiology; Center for Translational Research and Systems Medicine, } \\
\text { "Victor Babeş" University of Medicine and Pharmacy Timișoara } \\
\text { Privistirescu, Andreea; University of Medicine and Pharmacy Victor Babes } \\
\text { Timisoara, Pathophysiology } \\
\text { Wolf, Adrian; University of Medicine and Pharmacy Victor Babes Timisoara, } \\
\text { Pathophysiology } \\
\text { Petrus, Alexandra; University of Medicine and Pharmacy Victor Babes } \\
\text { Timisoara, Pathophysiology } \\
\text { Dănilă, Maria; University of Medicine and Pharmacy Victor Babes } \\
\text { Timisoara, Pathophysiology; Center for Translational Research and } \\
\text { Systems Medicine, "Victor Babeş" University of Medicine and Pharmacy } \\
\text { Timișoara } \\
\text { Ratiu, Corina; University of Medicine and Pharmacy Victor Babes Timisoara, } \\
\text { Pathophysiology } \\
\text { Muntean, Danina; University of Medicine and Pharmacy Victor Babes } \\
\text { Timisoara, Pathophysiology; Center for Translational Research and } \\
\text { Systems Medicine, "Victor Babeş" University of Medicine and Pharmacy } \\
\text { Timișoara } \\
\text { Sturza, Adrian; University of Medicine and Pharmacy Victor Babes } \\
\text { Timisoara, Pathophysiology; Center for Translational Research and } \\
\text { Systems Medicine, "Victor Babeş" University of Medicine and Pharmacy } \\
\text { Timișoara }\end{array}$ \\
\hline $\begin{array}{r}\text { Is the invited manuscript for } \\
\text { consideration in a Special } \\
\text { Issue?: }\end{array}$ & IACS European Section 2016 \\
\hline Keyword: & $\begin{array}{l}\text { rat heart mitochondria, diabetes mellitus, methylene blue, mitochondrial } \\
\text { respiration, reactive oxygen species }\end{array}$ \\
\hline
\end{tabular}


SCHOLARONE $^{\text {'t }}$
Manuscripts

https://mc06.manuscriptcentral.com/cjpp-pubs 


\section{METHYLENE BLUE IMPROVES MITOCHONDRIAL RESPIRATION AND \\ DECREASES OXIDATIVE STRESS IN A SUBSTRATE-DEPENDENT MANNER IN \\ DIABETIC RAT HEARTS}

Oana M. Duicu ${ }^{1,2 *}$, Andreea Privistirescu ${ }^{1 *}$, Adrian Wolf $^{1}$, Alexandra Petruș ${ }^{1}$, Maria D. Dănilă $\breve{1,2}^{1,}$ Corina D. Rațiu ${ }^{1}$, Danina M. Muntean ${ }^{1,2}$, Adrian Sturza ${ }^{1,2}$

${ }^{1}$ Department of Functional Sciences - Pathophysiology, ${ }^{2}$ Center for Translational Research and Systems Medicine, “Victor Babeş” University of Medicine and Pharmacy Timișoara, Romania

Corresponding author:

Danina M. Muntean, MD, PhD

Department of Pathophysiology,

"Victor Babeș" University of Medicine and Pharmacy of Timişoara

2, Eftimie Murgu Sq., 300041 Timisoara, RO

Tel/Fax: +40-256-493085

E-mail: daninamuntean@,umft.ro

*These authors contributed equally to this work. 


\begin{abstract}
Diabetic cardiomyopathy has been systematically associated with compromised mitochondrial energetics and increased generation of reactive oxygen species (ROS) that underlie its progression to heart failure. Methylene blue is a redox-drug with reported protective effects mainly on brain mitochondria. The present study was purported to characterize the effects of acute administration of methylene blue on mitochondrial respiration, $\mathrm{H}_{2} \mathrm{O}_{2}$ production, and calcium sensitivity in rat heart mitochondria isolated from healthy and 2 months (streptozotocininduced) diabetic rats. Mitochondrial respiratory function was assessed by high-resolution respirometry. Hydrogen peroxide production and calcium retention capacity were measured spectrofluorimetrically. The addition of methylene blue $(0.1 \mu \mathrm{M})$ elicited an increase in oxygen consumption of mitochondria energized with complex I and II substrates in both normal and diseased mitochondria. Interestingly, methylene blue elicited a significant increase in $\mathrm{H}_{2} \mathrm{O}_{2}$ release in the presence of CI substrates (glutamate-malate), but had an opposite effect in mitochondria energized with CII substrate (succinate). No changes in the calcium retention capacity of healthy or diabetic mitochondria were found in the presence of methylene blue. In conclusion, in cardiac mitochondria isolated from diabetic and non-diabetic rat hearts, methylene blue improved respiratory function and elicited a dichotomic, substrate-dependent effect on ROS production.
\end{abstract}

Keywords: rat heart mitochondria, diabetes mellitus, methylene blue, mitochondrial respiration, reactive oxygen species. 


\section{Introduction}

Coronary heart disease (CHD) is the leading cause of mortality due to myocardial infarction and of morbidity due to heart failure. In the past decades, mitochondrial dysfunction have emerged in both conditions central effectors of the pathomechanisms underlying both acute and chronic cardiac diseases as well as potential therapeutic targets (Bulluck et al. 2016; Waczulikova et al. 2007). Diabetes mellitus (DM), acknowledged as the pandemy of our century, is responsible for the diabetic cardiomyopathy, defined as the association of left ventricular hypertrophy/remodeling with diastolic dysfunction that precedes the development of systolic dysfunction and may progress to heart failure (Duicu et al. 2015a). Doenst et al. reported a reduced respiratory capacity of isolated mitochondria in association with hypertrophy and impaired ejection fraction at 20 weeks in the rat model of transverse aortic constriction (TAC) and chronic pressure overload (Doenst et al. 2010). Interestingly, the occurrence of contractile dysfunction at 20 weeks was no longer related to the ROS production in this model; moreover, the maximal ROS production occurred at 6 weeks after TAC concomitant with the onset of diastolic dysfunction (Schwarzer et al. 2014). Similarly, in the rabbit model of pressure-overload hypertrophy, mitochondrial complex I and II dysfunction occurred independent of an increase in the oxidative stress during the transition from compensated left ventricular hypertrophy to failure (Griffiths et al. 2010). Conversely, in a rat model of right-ventricular failure induced by pulmonary arterial hypertension, an increase in complex II activity and related ROS production were reported in mitochondria isolated from the right (but not from the left) ventricle (Redout et al. 2007). Abnormal cardiac energy metabolism and mitochondrial dysfunction are key factors in diabetic cardiomyopathy (reviewed in Bugger and Abel 2010); specifically, mitochondrial electron transport chain (ETC) defects with secondary drop in ATP synthesis and increase in reactive oxygen species (ROS) generation (Bugger and Abel 2014) occur in both diabetic rodents 
(Vazquez et al. 2015) and humans (Anderson et al. 2009). Thus, protecting mitochondrial function in cardiac diseases and diabetes represents a research priority for the academic community worldwide (Murphy et al. 2016).

Methylthioninium chloride, also known as methylene blue (MB), has been systematically investigated for its beneficial effects mainly in the treatment of neurodegenerative disorders such as Alzheimer and Parkinson (Oz et al. 2009); collectively, MB is widely recognized as being neuroprotective both in chronic neurodegenerative diseases and acute brain injury (reviewed in Jiang and Duong 2016). MB was also reported to attenuate ischemia/reperfusion (I/R) injury (Kelner et al. 1988) and increase the survival rate following experimental cardiac arrest in association with decreased oxidative myocardial damage (Miclescu et al. 2006).

Because mitochondrial function depends on redox components, drugs with redox activity have been increasingly used as mitochondrial modulators to enhance energy production and decrease oxidative stress (Atamna et al. 2012). As a redox active agent, with a mild redox potential $(10 \mathrm{mV}), \mathrm{MB}$ is enabled to readily cycle between its oxidized and reduced form $\mathrm{MBH}_{2}$ (Atamna et al. 2012). It has been proposed that MB is reduced by NADH dehydrogenase of complex I and reoxidized at cytochrome c, thus acting as an alternative electron carrier between NADH and cytochrome c (Atamna et al. 2012). This improves mitochondrial function and provides a mechanism to bypass complexes I and III, known to be the major sites for electron leakage and ROS production (Watts et al. 2013). Importantly, the protective effect of MB follows the pharmacological principle of hormesis, with an optimal effect only at low doses. In line with this principle, we have recently demonstrated that in vitro administration of $\mathrm{MB}(0.1 \mu \mathrm{M})$ increased both oxygen consumption rate (OCR) and extracellular acidification rate (ECAR), the two parameters of oxidative phosphorylation (OXPHOS) and glycolysis being assessed by the help of the extracellular flux analyzer Seahorse XF 24e (Duicu et al. 2015b). Of note, all OCR- 
linked parameters and ECAR were decreased in a concentration-dependent manner when MB was administered in higher concentrations $(1,5$, and $10 \mu \mathrm{M})$, and were not influenced by the lowest concentration tested $(0.05 \mu \mathrm{M})$, respectively (Duicu et al. 2015b).

Since impairment of respiration and increased ROS production are the major pathomechanisms underlying the progression of diabetic cardiomyopathy towards heart failure, we thought to investigate whether $\mathrm{MB}$ could improve mitochondrial respiration and alleviate oxidative stress in the presence of diabetes mellitus.

\section{Materials and Methods}

Animals had free access to water and fed on the normal laboratory rat chow throughout the experiment period of 8 weeks. Rats were housed under standard conditions (fully ventilated cages, in a temperature-controlled environment of $22 \pm 2^{\circ} \mathrm{C}$ with a $12 / 12$ hour light/dark cycle). Experiments were conducted in accordance with all guidelines for animal ethics and followed the Canadian Council on Animal Care (CCAC) guidelines. The experimental protocol was reviewed and approved by the Ethics Committee of the University for Medicine and Pharmacy of Timisoara.

Experiments were performed in adult Sprague Dawley (SD) male rats with $(n=8)$ and without $(n=6)$ diabetes mellitus (DM). Diabetes was induced by a single injection of streptozotocin $(50 \mathrm{mg} / \mathrm{kg} \mathrm{STZ}, \mathrm{IP})$. Age-matched control rats received an equal volume of vehicle (0.01 M citrate buffer, $\mathrm{pH} 4.5)$. Rats with non-fasting blood glucose (NFBG) over $200 \mathrm{mg} / \mathrm{dl}$ were considered diabetic. Animals were sacrificed after 2 months, while NFBG and body weight were regularly monitored. 


\section{Isolation of Cardiac Mitochondria}

Rat heart mitochondria (RHM) were isolated by differential centrifugations at $4{ }^{\circ} \mathrm{C}$, according to a previously described method (Duicu et al. 2013). Briefly, ventricles were treated with subtilizin ( $5 \mathrm{mg} / \mathrm{g}$ of wet cardiac tissue) and homogenized with a tissue homogenizer (Glas-Col 099C K5424 CE). Tissue homogenate was further centrifuged (Rotina centrifuge 38R) for 10 minutes at $8500 \mathrm{~g}$; the pellet was resuspended and centrifuged for 10 minutes at $800 \mathrm{~g}$. Lastly, the supernatant was centrifuged at $8500 \mathrm{~g}$ for 10 minutes. The final pellet of mitochondria was resuspended in a glass homogenizer, kept on ice, and used within four hours in respiratory rates measurements. The mitochondrial protein concentration was further evaluated, according to the Biuret method (Gornall et al. 1949).

\section{High-Resolution Respirometry Studies}

The oxygen consumption was measured at $37^{\circ} \mathrm{C}$ with the Oxygraph-2k (Oroboros Instruments, Austria). The mitochondria $(0.1 \mathrm{mg}$ protein $/ \mathrm{ml})$ were incubated in $2 \mathrm{ml}$ of incubation medium containing $0.5 \mathrm{mM}$ EGTA, $3 \mathrm{mM} \mathrm{MgCl} 2 \cdot 6 \mathrm{H}_{2} \mathrm{O}, 60 \mathrm{mM}$ K-lactobionate, $20 \mathrm{mM}$ taurine, $10 \mathrm{mM}$ $\mathrm{KH}_{2} \mathrm{P0}_{4}, 20 \mathrm{mM}$ HEPES, $110 \mathrm{mM}$ sucrose, $1 \mathrm{~g} / \mathrm{l} \mathrm{BSA}$, essentially fatty acid free $+280 \mathrm{U} / \mathrm{ml}$ catalase lyophilized powder, 2,000-5,000 units/mg protein $\left(\mathrm{pH} 7.1,37^{\circ} \mathrm{C}\right)$. The SubstrateUncoupler-Inhibitor Titration (SUIT) protocol adapted from Duicu et. al (Duicu et al. 2013) was as follows: chamber A: $\mathrm{GM}_{\text {STATE } 2}+\mathrm{ADP}_{\text {OXPHOS }}+\mathrm{cyt} \mathrm{c}+\mathrm{Omy}_{\text {STATE } 4}+\mathrm{FCCP}_{\text {ETS }}+\mathrm{Ama}_{\text {ROX }}$ and chamber B: $\mathrm{S}(\mathrm{Rot})_{\text {STATE } 2}+\mathrm{ADP}_{\text {OXPHOS }}+\mathrm{cyt} \mathrm{c}+\mathrm{Omy}_{\text {STATE } 4}+\mathrm{FCCP}_{\text {ETS }}+\mathrm{Ama}_{\text {ROX }}$. The protocol consisted of the following steps and the corresponding respiratory states: 1) addition of $10 \mathrm{mM}$ glutamate/G and $2 \mathrm{mM}$ malate/M (CI substrates) and $10 \mathrm{mM}$ succinate/S (CII substrate) +0.5 $\mu \mathrm{M}$ rotenone /Rot (CI inhibitor): STATE 2; 2) addition of $5 \mathrm{mM}$ ADP as a measure of the 
maximal oxidative phosphorylation capacity: OXPHOS capacity; 3) addition of $10 \mu \mathrm{M}$ cytochrome c/cyt $\mathrm{c}$ to assess the intactness of the outer mitochondrial membrane; 4) inhibition of ATP synthase by oligomycin/Omy $(2 \mu \mathrm{g} / \mathrm{ml})$ : STATE 4); 5) FCCP titration (0.5 $\mu \mathrm{M}$ steps), to obtain uncoupled respiration and to evaluate the electron transport system (ETS) capacity, and 6) inhibition of respiration with $2.5 \mu \mathrm{M}$ antimycin A/Ama (Residual OXygen consumption): ROX state. Mitochondrial respiration was further corrected for oxygen flux due to instrumental background and ROX.

\section{Mitochondrial $\mathrm{H}_{2} \mathrm{O}_{2}$ Production Assessment}

Mitochondrial $\mathrm{H}_{2} \mathrm{O}_{2}$ production was measured using the Amplex Red (10 $\left.\mu \mathrm{M}\right)$ fluorescent marker, as previously described (Duicu et al. 2013). The excitation wavelength was set to $530 \mathrm{~nm}$ and the emission wavelength to $590 \mathrm{~nm}$. Mitochondria $(0.25 \mathrm{mg}$ protein $/ \mathrm{ml})$ were incubated in 2 $\mathrm{ml}$ incubation buffer $(250 \mathrm{ml}$ sucrose, $1 \mathrm{mM}$ EGTA, $1 \mathrm{mM}$ EDTA, $20 \mathrm{mM}$ Tris/ $\mathrm{HCl}$ and 1.5 $\mathrm{mg} / \mathrm{ml}$ defatted $\mathrm{BSA}, \mathrm{pH} 7.4)$ at $37^{\circ} \mathrm{C}$, supplemented with glutamate + malate (5 mM each) or succinate $(5 \mathrm{mM})+$ rotenone $(0.5 \mu \mathrm{M})$. The fluorescence signal was calibrated by adding known amounts of $\mathrm{H}_{2} \mathrm{O}_{2}$ to the incubation buffer in the presence of Amplex Red and horseradish peroxidase. At the beginning of each measurement, the background fluorescence was quantified, in the absence of mitochondria. Net fluorescence was then calculated by measuring the fluorescence variation in function of time, minus background and $\mathrm{H}_{2} \mathrm{O}_{2}$ production was expressed in pmol $\mathrm{H}_{2} \mathrm{O}_{2} / \mathrm{min} / \mathrm{mg}$ protein. Results were expressed as pmol $\mathrm{H}_{2} \mathrm{O}_{2} / \mathrm{min} / \mathrm{mg}$ proteins.

\section{Calcium Retention Capacity Measurement}

To assess the in vitro sensitivity to $\mathrm{Ca}^{2+}$-induced mPTP (mitochondrial permeability transition pore) opening, the calcium retention capacity (CRC) was measured according to a technique 
previously described (Duicu et al. 2013). Measurement of calcium uptake by fresh RHM (0.25 $\mathrm{mg}$ protein/ml) was performed at $37^{\circ} \mathrm{C}$ in $2 \mathrm{ml}$ incubation buffer $(150 \mathrm{mM}$ sucrose, $50 \mathrm{mM} \mathrm{KCl}$,

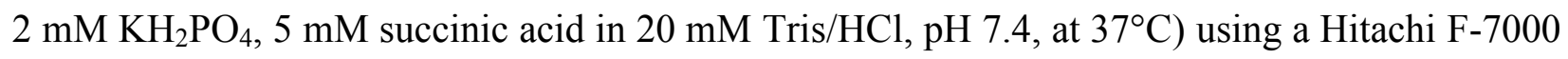
spectrofluorometer. The change in extramitochondrial $\mathrm{Ca}^{2+}$ concentration was monitored using the fluorescent probe Ca-green-5N (1 $\mu \mathrm{M}$; excitation-emission, $500-530 \mathrm{~nm}) . \mathrm{CaCl}_{2}$ pulses $(20$ $\mathrm{nmol} / \mathrm{pulse}$ ) were added every minute until a rapid increase of fluorescence occurred, signaling the opening of the mPTP. CRC was calculated as the cumulative amount of $\mathrm{Ca}^{2+}$ taken by mitochondria before $\mathrm{Ca}^{2+}$ release.

\section{Chemicals}

Calcium green-5N and Amplex Red were purchased from Invitrogen and cyclosporine A from Novartis, respectively. All the other chemicals were from Sigma-Aldrich.

\section{Statistical Analysis}

Data were expressed as means \pm SEM. Data analysis used one-way ANOVA followed by a posthoc Tukey test (GraphPad Prism v. 5.0 Software, SUA). Differences were considered statistically significant at $p<0.05$.

\section{Results and Discussions}

In diabetic animals, as expected, a reduced body weight and an elevated plasma glucose concentrations were measured (Table 1). 


\section{High-Resolution Respirometry Studies}

MB elicited a substrate-independent improvement in respiratory function as demonstrated by the increase in all respiratory parameters (i.e., State 2, OXPHOS, State 4, and ETS) in both non-diabetic (Fig. 1 A, B) and diabetic (Fig. 2 A, B) animals. Indeed, stimulation of respiration was observed for both Complex I and Complex II substrates, i.e., glutamate+malate and succinate ( + rotenone), respectively. These results are at variance from those reported in a recent study on isolated brain mitochondria which demonstrated that $\mathrm{MB}$ increased the rate of reduction of cytochrome $c$ when NADH was used as an electron donor, while, when the electron donor used was succinate, no significant increase in the rate of cytochrome $c$ reduction was observed (Lin et al. 2012). Similarly, Tretter et al. reported that, in mitochondria isolated from guinea-pig brain, MB stimulated only the oxygen consumption at rest and speculated that MB might not influence respiration of ATP synthesizing mitochondria in vivo, as it works in the presence of ADP all the time, in particular in the heart (Tretter et al. 2014).

In our hands, $\mathrm{MB}$ improved both basal and ADP-stimulated respiration, as shown by an increase in OXPHOS for both substrates, most probably by serving as an additional source of electrons for the ETS (Gonzalez-Lima and Auchter 2015): in non-diabetic group, MB increased OXPHOS by $29.8 \%$ in mitochondria energized with glutamate + malate and by $27.7 \%$ in mitochondria energized with succinate(+rotenone), while in diabetic group, MB increased OXPHOS by $24.4 \%$ in mitochondria energized with glutamate+malate and by $16 \%$ in mitochondria energized with succinate(+rotenone). The mild redox potential enables MB to cycle between its oxidized and reduced forms, providing a mechanism to shuttle electrons among 2 redox centers (i.e., oxidizing NADH via complex I and reducing cytochrome c) (Atamna et al. 2012). It has been hypothesized that, in the presence of $\mathrm{MBH}_{2}$ (with $\mathrm{NADH}$ as the major electron 
donor), cytochrome $\mathrm{c}$ is reduced above the normal enzymatic reduction by complex III leading to increased cytochrome oxidase (complex IV) activity and subsequent oxygen consumption (Atamna et al. 2012).

In line with the previously mentioned hormetic behavior, it is important to emphasize that the beneficial effects of $\mathrm{MB}$ were observed at very low doses (i.e., $0.1 \mu \mathrm{M}$ ). In high concentrations, it has been speculated that MB could "steal" electrons from the ETC complexes resulting in a disrupted redox balance (Rojas et al. 2012). Accordingly, Tretter and colleagues also remarked that $\mathrm{MB}$ partially restored respiration if electron flux was inhibited at either complex I or III (Tretter et al. 2014). This might be of particular importance in I/R injury where a massive release of ROS damages various cellular structures, including mitochondrial respiratory complexes, contributing thus to diminished metabolic activity and increased ROS production.

Our data also demonstrated that experimental diabetes was associated with mitochondrial respiratory dysfunction, as previously reported in chronic (Bugger and Abel 2010; Bugger and Abel 2014), as well as in acute diabetic heart (Ferko et al. 2006). Accordingly, in diabetic RHM respiring on glutamate+malate, we found a significant decrease in all bioenergetic parameters $v s$. the non-diabetic group, as follows: in untreated controls: STATE $2(41.6 \pm 4.9$ vs. $44.9 \pm 3.8$ pmol.s-1.ml-1, $p<0.05)$, OXPHOS (450.5 \pm 34.4 vs. $750.7 \pm 68.4$ pmol.s-1.ml-1, $p<0.01)$, STATE 4 (71.1 \pm 7.4 vs. 93.6 \pm 5.2 pmol.s-1.ml-1, $p<0.05)$, and ETS (420.4 \pm 48 vs. $681.1 \pm 39.1$ pmol.s-1.ml-1, $p<0.01)$; in controls treated with MB: STATE 2 (70.45 \pm 4.6 vs. 66.2 \pm 3.2 pmol.s-1.ml-1, $p<0.05)$,

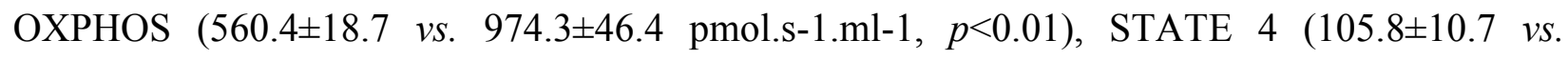
119.6 \pm 6 pmol.s-1.ml-1, $p<0.05)$, and ETS (614.7 $\pm 67.6 v s .842 .9 \pm 46.2$ pmol.s-1.ml-1, $p<0.05)$. A comparable respiratory impairment of diabetic mitochondria was recorded in the presence of

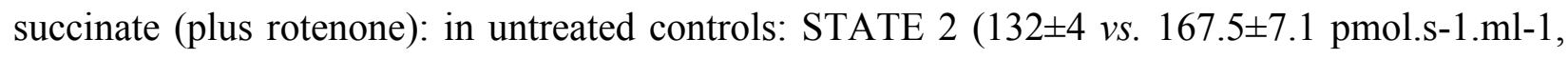
$p<0.05)$, OXPHOS (567.6 $\pm 11.2 v s .708 .2 \pm 37.1$ pmol.s-1.ml-1, $p<0.01)$, STATE 4 (267 $\pm 33.1 v s$. 
301 \pm 14.6 pmol.s-1.ml-1, $p<0.05$ ), and ETS (643.2 \pm 11.2 vs. 741 \pm 40.3 pmol.s-1.ml-1, $p<0.01)$,

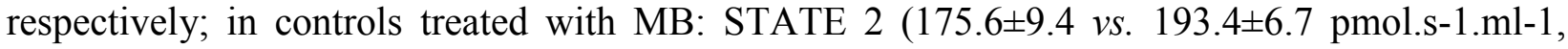
$p<0.05)$, OXPHOS (658.7 \pm 30 vs. 904.1 \pm 32.9 pmol.s-1.ml-1, $p<0.01)$, STATE 4 (393.3 $\pm 36.3 v s$. 48.3 \pm 1.9 pmol.s-1.ml-1, $p<0.05$ ), and ETS (744 \pm 23.7 vs. 873.1 \pm 18.8 pmol.s-1.ml-1, $p<0.01)$.

\section{Mitochondrial $\mathrm{H}_{2} \mathrm{O}_{2}$ Production Assessment}

The effects of $\mathrm{MB}(0.1 \mu \mathrm{M})$ on ROS production were further assessed in RHM respiring either on complex I or complex II substrates, using the Amplex Red technique. Rotenone (complex I inhibitor) was used in combination with succinate to prevent oxaloacetate accumulation, which is a strong competitive inhibitor of succinate dehydrogenase; additionally it prevents the reverse electron transport (RET) from complex II to I, known as a major ROS producing-process during succinate oxidation.

As expected (Bugger and Abel 2010; Bugger and Abel 2014), in diabetic RHM we found a significant increase in $\mathrm{H}_{2} \mathrm{O}_{2}$ production vs. the non-diabetic animals in the presence of both glutamate+malate (in untreated controls: $19.6 \pm 3.5$ vs. $14.8 \pm 2.4 \mathrm{pmol} / \mathrm{mg} \mathrm{prot} / \mathrm{min}, p<0.5$; in controls treated with MB: $61 \pm 3.7$ vs. $26.3 \pm 4 \quad \mathrm{pmol} / \mathrm{mg}$ prot $/ \mathrm{min}, \quad p<0.01$ ) and succinate(+rotenone) (in untreated controls: $159 \pm 6.8$ vs. $87.25 \pm 14.8 \mathrm{pmol} / \mathrm{mg} \mathrm{prot} / \mathrm{min}, p<0.01$; in controls treated with MB: $81.1 \pm 4.8 v s .40 .3 \pm 9.6 \mathrm{pmol} / \mathrm{mg} \mathrm{prot} / \mathrm{min}, p<0.01)$.

Interestingly, the addition of $\mathrm{MB}(0.1 \mu \mathrm{M})$ significantly increased $\mathrm{H}_{2} \mathrm{O}_{2}$ production in both groups (with/without diabetes) when mitochondria were energized with glutamate+malate (in diabetic group by $210 \%$, and in non-diabetic group by $78 \%$ ), whereas a significant decrease was observed when succinate (+rotenone) was used as substrate (in diabetic group by $49 \%$, and in non-diabetic group by 53.8\%) (Fig. 3 A, B). 
The former result regarding mitochondrial $\mathrm{H}_{2} \mathrm{O}_{2}$ production was rather surprising view the antioxidant effect of MB described in the literature (Ryou et al. 2015; Poteet et al. 2012). There is only one study in the literature (by Tretter et al.) that reported an enhanced $\mathrm{H}_{2} \mathrm{O}_{2}$ production with $\mathrm{MB}$ in isolated guinea pig brain mitochondria respiring on both glutamate+malate and succinate+rotenone (Tretter et al. 2014). These authors suggested that, besides cytochrome c, MB could directly reduce $\mathrm{O}_{2}$, thus generating $\mathrm{H}_{2} \mathrm{O}_{2}$. As $\mathrm{MB}$ preferentially reduces cytochrome c over $\mathrm{O}_{2}$, Atamna et al. hypothesized that cytochrome c levels become limited in the presence of high concentrations of $\mathrm{MBH}_{2}$ (Atamna et al. 2008). In this view, they suggested that a high ratio between cytochrome $\mathrm{c}$ and $\mathrm{MB}$ is fundamental in order to prevent autooxidation of $\mathrm{MBH}_{2}$, since an excessive cytochrome $\mathrm{c}$ addition to the mitochondrial lysate, NADH, rotenone, and $\mathrm{MB}$ significantly eliminated the production of oxidants. (Atamna et al. 2008) According to Gnaiger, in rat heart mitochondria, the oxidation of succinate (+rotenone) is marginally higher compared to glutamate+malate (Gnaiger 2014). As a consequence, a saturation of cytochrome c and the subsequent enhance in $\mathrm{H}_{2} \mathrm{O}_{2}$ production should have more likely occurred with the complex II substrate (and not with complex I substrates), indicating that direct reduction of $\mathrm{O}_{2}$ by $\mathrm{MBH}_{2}$ to form ROS cannot explain our observations. As MB accelerates the oxidation of NADH by serving as an additional electron acceptor, a smaller $\mathrm{NADH} / \mathrm{NAD}^{+}$ratio is expected, therefore excluding this site as a cause for enhanced ROS production with glutamate+malate as substrate (Rojas et al. 2012). In addition to the commonly accepted concept that MB donates its electrons to cytochrome c and downstream, Rojas and colleagues also consider coenzyme Q as a putative MB target (Rojas et al. 2012). In this respect, as MB independently supplies electrons to the coenzyme Q pool, this could allow the occurrence of RET in mitochondria respiring on glutamate+malate. The second site at complex I, the CoQ-binding domain 1Q, is of particular 
importance during succinate-supported respiration, as it constitutes the major locus for ROS generation during RET. While $1 \mathrm{Q}$ is negligible during regular forward electron transport with glutamate+malate, it could constitute a major ROS-generating site in the presence of $\mathrm{MB}$. This hypothesis is supported by the work of Muller et al. who reported high superoxide production in mitochondria respiring on glutamate + succinate, that was decreased by rotenone and completely abolished by the uncoupler FCCP, indicating RET as a cause (Muller et al. 2007). It was concluded that RET mediated superoxide production can occur under physiological realistic substrate conditions, but oxaloacetate inhibition of complex II is meant to minimize that (Muller et al. 2007). We cannot exclude the fact that RET might occur during physiological, complex Isupported respiration and that $\mathrm{MB}$, independently of oxaloacetate inhibition, could deliver electrons to the coenzyme Q pool; in these conditions $1 \mathrm{Q}$ site might become the potential candidate able to explain the increased ROS production that we observed with glutamate+malate.

Nevertheless, we admit as important limitation of our study the fact that we did not measure the mitochondrial membrane potential, especially since Waczulikova et al. demonstrated a slight decline of the membrane potential concomitant with an increased membrane fluidity in acute (i.e., 8 days) streptozotocin-induced diabetic rat hearts (Waczulikova et al. 2007). Membrane potential is thought to slow down electron transfer between heme $b_{\mathrm{L}}$ and heme $b_{\mathrm{H}}$ thus supporting a more reduced heme $b_{\mathrm{L}}$ which then increases ROS production (Bleier and Drose 2013). If we apply this model on MB and hypothesize that, despite some uncoupling action, membrane potential was increased with both substrates, leading to a more reduced heme $b_{\mathrm{L}}$, the main determinant of ROS production would be the redox state of coenzyme Q pool. Dröse and Bandt showed in submitochondrial particles fueled with succinate that an increased ROS production from complex III occurred with the inhibition of succinate dehydrogenase (SDH) to a certain threshold (Drose and Brandt 2008). Accordingly, we can reverse their observation and 
hypothesize that SDH activity is enhanced by MB and by thus, ROS production will decrease. If $\mathrm{MBH}_{2}$ donates electrons in addition to those fed by complex I or II to coenzyme Q (Rojas et al. 2012), than ROS production will decrease with the stronger substrate, i.e. succinate, that produced high amounts of ROS before the addition of MB at „optimal“ reduced coenzyme Q pool, as it maximally reduces coenzyme Q leaving no oxidized forms to generate the new intermediate; thereby ROS production might increase at Qo in the presence of the weaker substrates glutamate+malate, as $\mathrm{MBH}_{2}$ just gives the additional Q pool reduction needed for maximal production. If MB did not change the membrane potential, but at least maintained it, this mechanism could still provide a reasonable good explanation for our observations. A study by Quinlan and colleagues conducted on mitochondria isolated from rat hind limb skeletal muscles showed that in the absence of antimycin A (CIII inhibitor), succinate+rotenone produced approximately twice the amount of ROS at Qo as compared to glutamate+malate (Quinlan et al. 2013); these results suggest that an over reduction of coenzyme Q pool might indeed have led to changes observed in our experiments.

\section{Calcium Retention Capacity (CRC) Measurement}

The in vitro capability of mitochondria to uptake calcium prior the opening of the mitochondrial permeability pore ( $\mathrm{MPTP})$ or the calcium retention capacity $(\mathrm{CRC})$ was assessed in isolated RHM in the presence $v s$. the absence of the classical pore desensitizer, cyclosporine A (CsA). As expected, with CsA we observed a decreased sensitivity to $\mathrm{Ca}^{2+}$-induced mPTP opening vs. untreated controls (Fig. 4). 
It is already well established that prolonged calcium transients occur in both acute and chronic diabetic heart; in this view, Ziegelhoffer et al. demonstrated the presence of a strong calcium signaling in the acute phase of streptozotocin-induced diabetes (Ziegelhoffer et al. 2009). In our study, a significant decrease in CRC was found, as expected, in diabetic vs. non-diabetic mitochondria regardless the absence or the presence of CsA: in untreated controls: $613 \pm 230 v s$. $824 \pm 118.7 \mathrm{nmol} \mathrm{CaCl}_{2} / \mathrm{mg}$ prot $(p<0.05)$; in controls treated with MB: $666.7 \pm 74.5$ vs. $780 \pm 47.3$ $\mathrm{nmol} \mathrm{CaCl}_{2} / \mathrm{mg}$ prot $(p<0.05)$; in controls treated with CsA: $1200 \pm 312$ vs. $1783 \pm 312.3 \mathrm{nmol}$ $\mathrm{CaCl}_{2} / \mathrm{mg}$ prot $(p<0.05)$. These results are suggestive for the increased propensity of the diabetic hearts to undergo the phenomenon of permeability transition.

However, the addition of MB in either group (diabetic and non-diabetic) of mitochondria did not interfere with the capability of mitochondria to buffer calcium (Fig. 5). The rationale behind this assay was to estimate whether the bioenergetic changes induced by MB could desensitize mPTP for calcium overload, since there are numerous factors able to modulate the phenomenon of permeability transition by changing the sensitivity of mPTP to calcium ions (Halestrap and Richardson 2015).

Even though MB did not change sensitivity to $\mathrm{Ca}^{2+}$-induced $\mathrm{mPTP}$ opening in respiring mitochondria, whether this will be the case in mitochondria isolated at the postischemic reperfusion remains to be determined.

\section{Conclusions}

In conclusion, acute in vitro administration of methylene blue, in very low doses, improved mitochondrial respiration and elicited a dichotomic, substrate-dependent effect on ROS production in diabetes that clearly warrants further investigation in line with the highly-favored concept of drug repurposing. 


\section{Conflicts of interest}

There is no conflict of interest.

OR

\section{Disclosures:}

None

\section{References}

Anderson, E.J., Kypson, A.P., Rodriguez, E., Anderson, C.A., Lehr, E.J., and Neufer, P.D. 2009. Substrate-specific derangements in mitochondrial metabolism and redox balance in the atrium of the type 2 diabetic human heart. Journal of the American College of Cardiology 54(20): 18911898. doi: 10.1016/j.jacc.2009.07.031.

Atamna, H., Mackey, J., and Dhahbi, J.M. 2012. Mitochondrial pharmacology: electron transport chain bypass as strategies to treat mitochondrial dysfunction. BioFactors 38(2): 158-166. doi: 10.1002/biof.197.

Atamna, H., Nguyen, A., Schultz, C., Boyle, K., Newberry, J., Kato, H., and Ames, B.N. 2008. Methylene blue delays cellular senescence and enhances key mitochondrial biochemical pathways. FASEB journal : official publication of the Federation of American Societies for Experimental Biology 22(3): 703-712. doi: 10.1096/fj.07-9610com.

Bleier, L., and Drose, S. 2013. Superoxide generation by complex III: from mechanistic rationales to functional consequences. Biochimica et biophysica acta 1827(11-12): 1320-1331. doi: 10.1016/j.bbabio.2012.12.002.

Bugger, H., and Abel, E.D. 2010. Mitochondria in the diabetic heart. Cardiovascular research 88(2): 229-240. doi: 10.1093/cvr/cvq239. 
Bugger, H., and Abel, E.D. 2014. Molecular mechanisms of diabetic cardiomyopathy. Diabetologia 57(4): 660-671. doi: 10.1007/s00125-014-3171-6.

Bulluck, H., Yellon, D.M., and Hausenloy, D.J. 2016. Reducing myocardial infarct size: challenges and future opportunities. Heart 102(5): 341-348. doi: 10.1136/heartjnl-2015-307855.

Doenst, T., Pytel, G., Schrepper, A., Amorim, P., Farber, G., Shingu, Y., Mohr, F.W., and Schwarzer, M. 2010. Decreased rates of substrate oxidation ex vivo predict the onset of heart failure and contractile dysfunction in rats with pressure overload. Cardiovascular research 86(3): 461-470. doi: 10.1093/cvr/cvp414.

Drose, S., and Brandt, U. 2008. The mechanism of mitochondrial superoxide production by the cytochrome bcl complex. The Journal of biological chemistry 283(31): 21649-21654. doi: 10.1074/jbc.M803236200.

Duicu, O.M., Lighezan, R., Sturza, A., Ceausu, R.A., Borza, C., Vaduva, A., Noveanu, L., Gaspar, M., Ionac, A., Feier, H., Muntean, D.M., and Mornos, C. 2015a. Monoamine Oxidases as Potential Contributors to Oxidative Stress in Diabetes: Time for a Study in Patients Undergoing Heart Surgery. BioMed research international 2015: 515437. doi: 10.1155/2015/515437.

Duicu, O.M., Mirica, S.N., Gheorgheosu, D.E., Privistirescu, A.I., Fira-Mladinescu, O., and Muntean, D.M. 2013. Ageing-induced decrease in cardiac mitochondrial function in healthy rats. Canadian journal of physiology and pharmacology 91(8): 593-600. doi: 10.1139/cjpp-2012-0422. Duicu, O.M., Scurtu, I., Popescu, R., Sturza, A., Coricovac, D., Danila, D.M., Privistirescu, A., and Muntean, M.D. 2015b. Assessment of the effects of methylene blue on cellular bioenergetics in H9c2 cells. Rev Chim (Bucharest) 2015 66(4): 519-522.

Ferko, M., Gvozdjakova, A., Kucharska, J., Mujkosova, J., Waczulikova, I., Styk, J., Ravingerova, T., Ziegelhoffer-Mihalovicova, B., and Ziegelhoffer, A. 2006. Functional 
remodeling of heart mitochondria in acute diabetes: interrelationships between damage, endogenous protection and adaptation. General physiology and biophysics 25(4): 397-413.

Gnaiger, E. 2014. Mitochondrial Pathways and Respiratory Control An Introduction to OXPHOS Analysis. Mitochondr Physiol Network 19.12. OROBOROS MiPNet Publications, Innsbruck 4th ed.: 80 .

Gonzalez-Lima, F., and Auchter, A. 2015. Protection against neurodegeneration with low-dose methylene blue and near-infrared light. Frontiers in Cellular Neuroscience 9: 179. doi: 10.3389/fncel.2015.00179.

Gornall, A.G., Bardawill, C.J., and David, M.M. 1949. DETERMINATION OF SERUM PROTEINS BY MEANS OF THE BIURET REACTION. Journal of Biological Chemistry 177(2): 751-766.

Griffiths, E.R., Friehs, I., Scherr, E., Poutias, D., McGowan, F.X., and Del Nido, P.J. 2010. Electron transport chain dysfunction in neonatal pressure-overload hypertrophy precedes cardiomyocyte apoptosis independent of oxidative stress. The Journal of thoracic and cardiovascular surgery 139(6): 1609-1617. doi: 10.1016/j.jtcvs.2009.08.060.

Halestrap, A.P., and Richardson, A.P. 2015. The mitochondrial permeability transition: a current perspective on its identity and role in ischaemia/reperfusion injury. Journal of molecular and cellular cardiology 78: 129-141. doi: 10.1016/j.yjmcc.2014.08.018.

Jiang, Z., and Duong, T.Q. 2016. Methylene blue treatment in experimental ischemic stroke: a mini review. Brain circulation 2(1): 48-53. doi: 10.4103/2394-8108.178548.

Kelner, M.J., Bagnell, R., Hale, B., and Alexander, N.M. 1988. Potential of Methylene Blue to Block Oxygen Radical Generation in Reperfusion Injury. In Oxygen Radicals in Biology and Medicine. Edited by M.G. Simic and K.A. Taylor and J.F. Ward and C. von Sonntag. Springer US, Boston, MA. pp. 895-898. 
Lin, A.L., Poteet, E., Du, F., Gourav, R.C., Liu, R., Wen, Y., Bresnen, A., Huang, S., Fox, P.T., Yang, S.H., and Duong, T.Q. 2012. Methylene blue as a cerebral metabolic and hemodynamic enhancer. PloS one 7(10): e46585. doi: 10.1371/journal.pone.0046585.

Miclescu, A., Basu, S., and Wiklund, L. 2006. Methylene blue added to a hypertonichyperoncotic solution increases short-term survival in experimental cardiac arrest. Critical care medicine 34(11): 2806-2813. doi: 10.1097/01.ccm.0000242517.23324.27.

Muller, Florian L., Liu, Y., Abdul-Ghani, Muhammad A., Lustgarten, Michael S., Bhattacharya, A., Jang, Youngmok C., and Van Remmen, H. 2007. High rates of superoxide production in skeletal-muscle mitochondria respiring on both complex I- and complex II-linked substrates. Biochemical Journal 409(2): 491.

Murphy, E., Ardehali, H., Balaban, R.S., DiLisa, F., Dorn, G.W., 2nd, Kitsis, R.N., Otsu, K., Ping, P., Rizzuto, R., Sack, M.N., Wallace, D., and Youle, R.J. 2016. Mitochondrial Function, Biology, and Role in Disease: A Scientific Statement From the American Heart Association. Circulation research 118(12): 1960-1991. doi: 10.1161/res.0000000000000104.

Oz, M., Lorke, D.E., and Petroianu, G.A. 2009. Methylene blue and Alzheimer's disease. Biochemical Pharmacology 78(8): 927-932. doi: http://dx.doi.org/10.1016/j.bcp.2009.04.034.

Poteet, E., Winters, A., Yan, L.J., Shufelt, K., Green, K.N., Simpkins, J.W., Wen, Y., and Yang, S.H. 2012. Neuroprotective actions of methylene blue and its derivatives. PloS one 7(10): e48279. doi: 10.1371/journal.pone.0048279.

Quinlan, C.L., Perevoshchikova, I.V., Hey-Mogensen, M., Orr, A.L., and Brand, M.D. 2013. Sites of reactive oxygen species generation by mitochondria oxidizing different substrates. Redox biology 1(1): 304-312. doi: http://dx.doi.org/10.1016/i.redox.2013.04.005.

Redout, E.M., Wagner, M.J., Zuidwijk, M.J., Boer, C., Musters, R.J., van Hardeveld, C., Paulus, W.J., and Simonides, W.S. 2007. Right-ventricular failure is associated with increased 
mitochondrial complex II activity and production of reactive oxygen species. Cardiovascular research 75(4): 770-781. doi: 10.1016/j.cardiores.2007.05.012.

Rojas, J.C., Bruchey, A.K., and Gonzalez-Lima, F. 2012. Neurometabolic mechanisms for memory enhancement and neuroprotection of methylene blue. Progress in neurobiology 96(1): 32-45. doi: 10.1016/j.pneurobio.2011.10.007.

Ryou, M.-G., Choudhury, G.R., Li, W., Winters, A., Yuan, F., Liu, R., and Yang, S.-H. 2015. Methylene blue-induced neuronal protective mechanism against hypoxiareoxygenation stress. Neuroscience 301: 193-203. doi: 10.1016/j.neuroscience.2015.05.064.

Schwarzer, M., Osterholt, M., Lunkenbein, A., Schrepper, A., Amorim, P., and Doenst, T. 2014. Mitochondrial ROS production and respiratory complex activity in rats with pressure overloadinduced heart failure. The Journal of physiology. doi: 10.1113/jphysiol.2014.274704.

Tretter, L., Horvath, G., Hölgyesi, A., Essek, F., and Adam-Vizi, V. 2014. Enhanced hydrogen peroxide generation accompanies the beneficial bioenergetic effects of methylene blue in isolated brain mitochondria. Free Radical Biology and Medicine 77: 317-330. doi: http://dx.doi.org/10.1016/j.freeradbiomed.2014.09.024.

Vazquez, E.J., Berthiaume, J.M., Kamath, V., Achike, O., Buchanan, E., Montano, M.M., Chandler, M.P., Miyagi, M., and Rosca, M.G. 2015. Mitochondrial complex I defect and increased fatty acid oxidation enhance protein lysine acetylation in the diabetic heart. Cardiovascular research 107(4): 453-465. doi: 10.1093/cvr/cvv183.

Waczulikova, I., Habodaszova, D., Cagalinec, M., Ferko, M., Ulicna, O., Mateasik, A., Sikurova, L., and Ziegelhoffer, A. 2007. Mitochondrial membrane fluidity, potential, and calcium transients in the myocardium from acute diabetic rats. Canadian journal of physiology and pharmacology 85(3-4): 372-381. doi: 10.1139/y07-035. 
Watts, L.T., Lloyd, R., Garling, R.J., and Duong, T. 2013. Stroke neuroprotection: targeting mitochondria. Brain sciences 3(2): 540-560. doi: 10.3390/brainsci3020540.

Ziegelhoffer, A., Waczulikova, I., Ferko, M., Kincelova, D., Ziegelhoffer, B., Ravingerova, T., Cagalinec, M., Schonburg, M., Ziegelhoeffer, T., Sikurova, L., Ulicna, O., and Mujkosova, J. 2009. Calcium signaling-mediated endogenous protection of cell energetics in the acutely diabetic myocardium. Canadian journal of physiology and pharmacology 87(12): 1083-1094. doi: 10.1139/y09-108.

\section{TABLES}

Table 1. Animal weights and biochemical characteristics in the studied groups.

\begin{tabular}{|l|c|c|}
\hline & CTRL & DM \\
\hline Weight (grams) & & \\
Time 0 & $513.4 \pm 13.8$ & $521.7 \pm 24.8$ \\
After 2 months & $589.0 \pm 25.9$ & $382.5 \pm 49.5 *$ \\
\hline NFBG (mg/dL) & $104.5 \pm 4.4$ & $373.2 \pm 94.4 *$ \\
\hline
\end{tabular}

Note. $\mathrm{NFBG}=$ non-fasting blood glucose. Values are means \pm SEM. $*: p<0.05 v s$. CTRL. 


\section{FIGURES CAPTIONS}

Fig. 1. High-resolution respirometry data for CI-supported respiration. (A) CTRL group; (B) DM group. Values are means \pm S.E.M. ${ }^{*} p<0.05 ; * *<<0.01$.

Fig. 2. High-resolution respirometry data for CII-supported respiration. (A) CTRL group; (B) DM group. Values are means \pm S.E.M. ${ }^{*} p<0.05,{ }^{*} p<00.01$.

Fig. 3. Mitochondrial $\mathrm{H}_{2} \mathrm{O}_{2}$ production in CTRL (A) and DM (B) groups. Additions are as follows: A1, B1: GM (5 mM glutamate $+5 \mathrm{mM}$ malate), and A2, B2: SRot (5 mM succinate + $0.5 \mu \mathrm{M}$ rotenone). Values are means $\pm \mathrm{SEM} . * p<0.05, * * p<0.01$

Fig. 4. Calcium retention capacity assay: representative traces of $\mathrm{CaCl}_{2}$ pulses without and with $1 \mu \mathrm{M}$ CsA in RHM from CTRL group. $\mathrm{CaCl}_{2}$ pulses $(20 \mathrm{nmol} /$ pulse $)$ were added at $1 \mathrm{~min}$ intervals, until mitochondrial $\mathrm{Ca}^{2+}$ release caused by opening of the PTP was observed.

Fig. 5. Calcium retention capacity data in CTRL (A) and DM (B) rats. Values are means \pm SEM. ${ }^{*} p<0.05, * * p<0.01$ 
A.
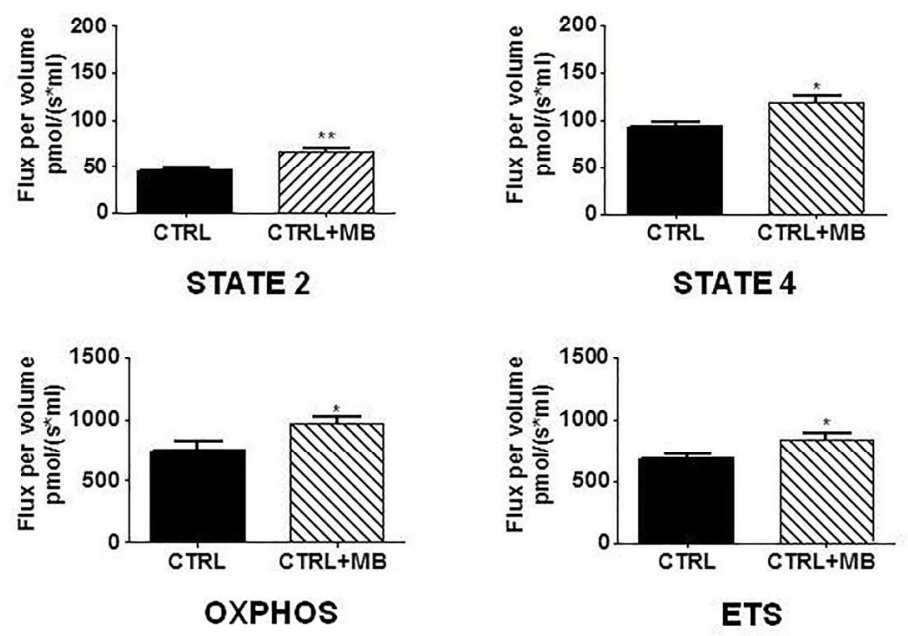

B.
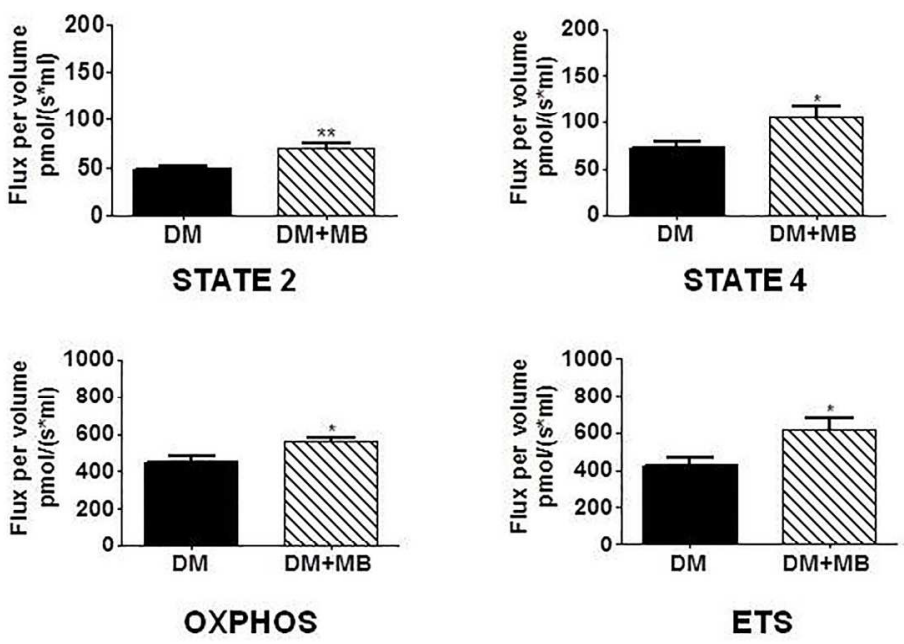

Fig. 1. High-resolution respirometry data for CI-supported respiration. (A) CTRL group; (B) DM group. Values are means \pm S.E.M. ${ }^{*} p<0.05 ; * * p<0.01$

$190 \times 254 \mathrm{~mm}(300 \times 300 \mathrm{DPI})$ 
A.
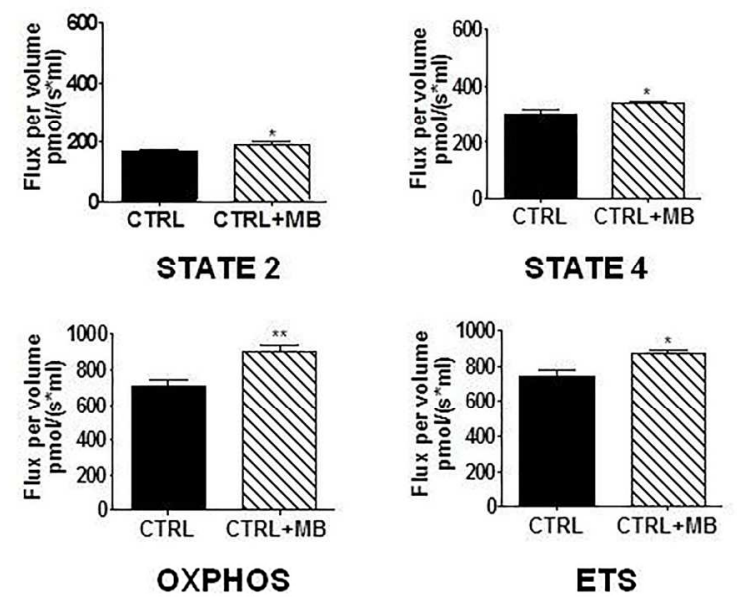

B.
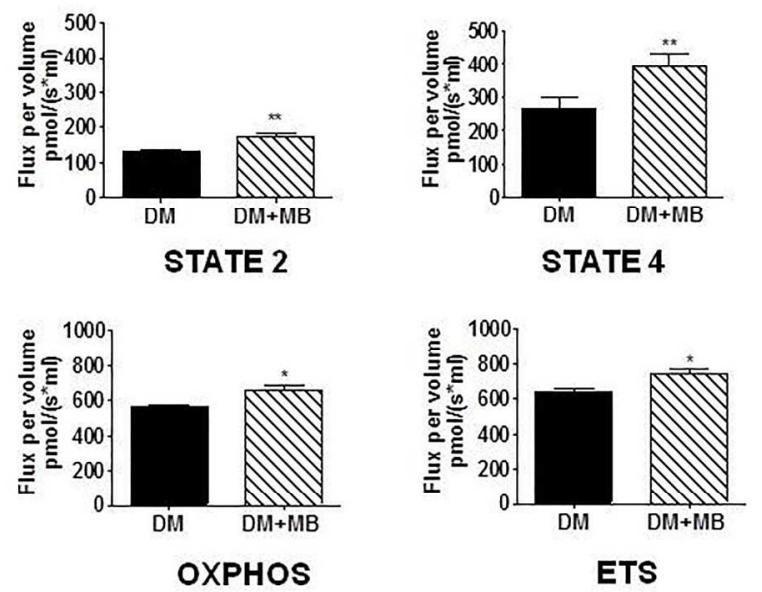

Fig. 2. High-resolution respirometry data for CII-supported respiration. (A) CTRL group; (B) DM group. Values are means \pm S.E.M. $* p<0.05, * * p<0.01$

$190 \times 254 \mathrm{~mm}(300 \times 300 \mathrm{DPI})$ 
A.1.

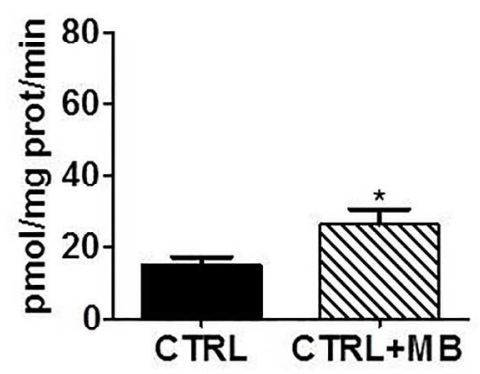

B.1.

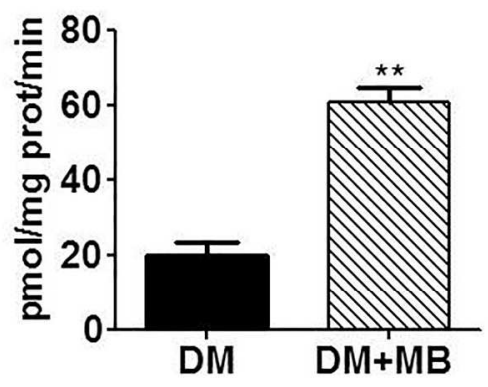

A.2.

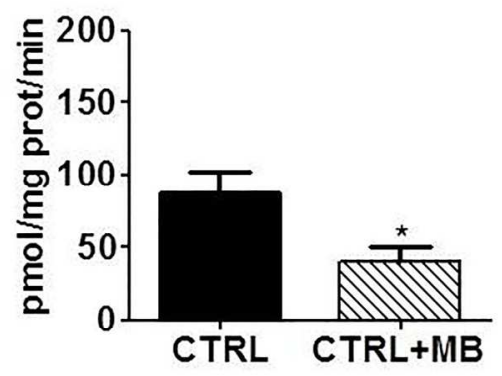

B.2.

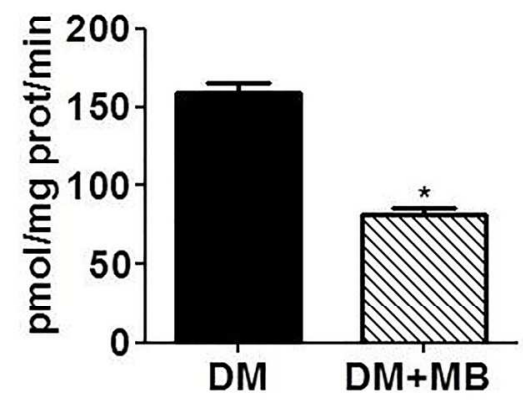

Fig. 3. Mitochondrial $\mathrm{H} 2 \mathrm{O} 2$ production in $\mathrm{CTRL}(\mathrm{A})$ and $\mathrm{DM}(\mathrm{B})$ groups. Additions are as follows: $\mathrm{A} 1, \mathrm{B1}$ : GM ( $5 \mathrm{mM}$ glutamate $+5 \mathrm{mM}$ malate), and A2, B2: SRot ( $5 \mathrm{mM}$ succinate $+0.5 \mu \mathrm{M}$ rotenone). Values are means \pm SEM. $* \mathrm{p}<0.05, * * \mathrm{p}<0.01$.

$190 \times 254 \mathrm{~mm}(300 \times 300 \mathrm{DPI})$ 


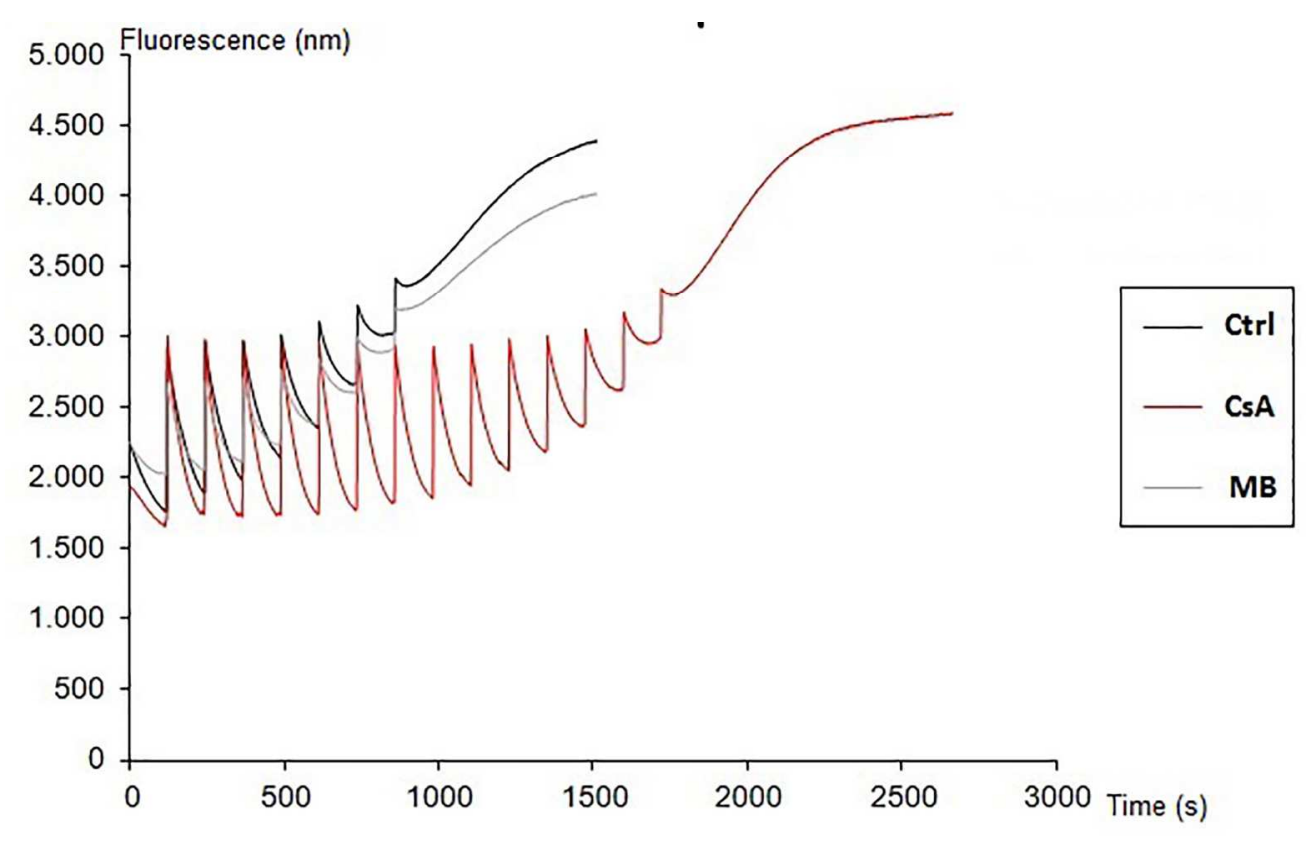

Fig. 4. Calcium retention capacity assay: representative traces of $\mathrm{CaCl} 2$ pulses without and with $1 \mu \mathrm{M}$ CsA in RHM from CTRL group. CaCl2 pulses ( $20 \mathrm{nmol} /$ pulse) were added at 1 min intervals, until mitochondrial $\mathrm{Ca} 2+$ release caused by opening of the PTP was observed.

$161 \times 100 \mathrm{~mm}(300 \times 300 \mathrm{DPI})$ 
A.

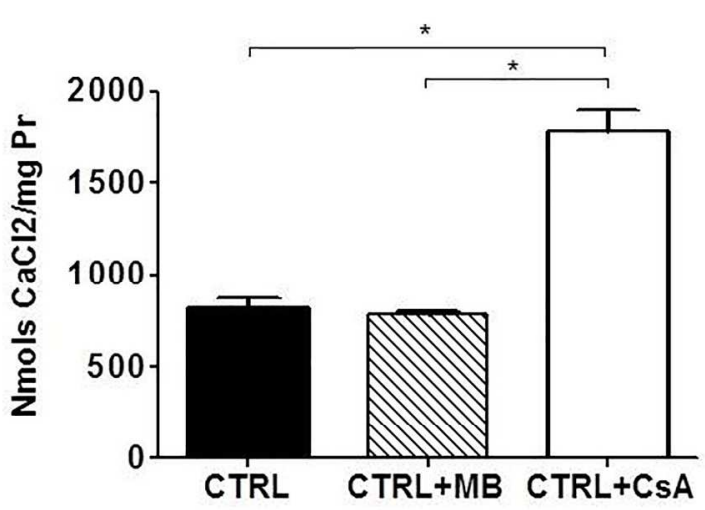

B.

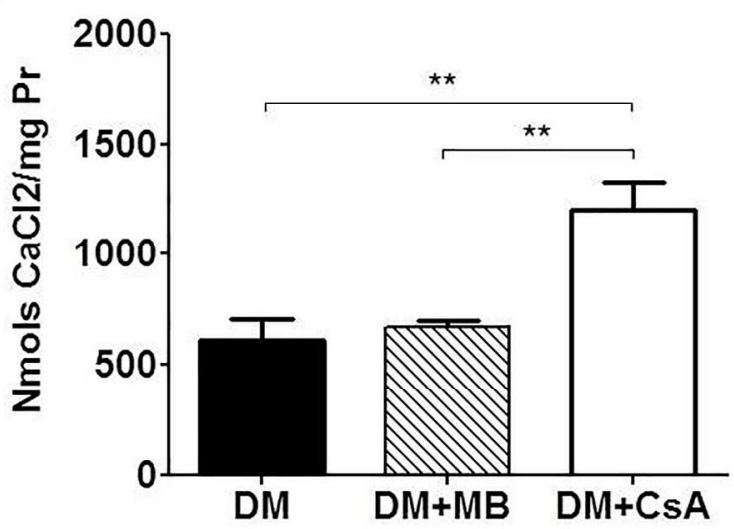

Fig. 5. Calcium retention capacity data in $\operatorname{CTRL}(A)$ and $D M(B)$ rats. Values are means $\pm S E M . * p<0.05$, $* * \mathrm{p}<0.01$.

$190 \times 254 \mathrm{~mm}(300 \times 300 \mathrm{DPI})$ 